\title{
Exact and explicit solutions to nonlinear evolution equations using the division theorem
}

\author{
İsmail Aslan \\ Department of Mathematics, Izmir Institute of Technology, Urla, Izmir 35430, Turkey
}

\section{A R T I C L E I N F O}

\section{Keywords:}

First integral method

Regularized long-wave Burgers equation

Gilson-Pickering equation

Traveling wave solutions

\begin{abstract}
A B S T R A C T
In this paper, we show the applicability of the first integral method, which is based on the ring theory of commutative algebra, to the regularized long-wave Burgers equation and the Gilson-Pickering equation under a parameter condition. Our method provides polynomial first integrals for autonomous planar systems. Through the established first integrals, exact traveling wave solutions are derived in a concise manner.
\end{abstract}

(c) 2011 Elsevier Inc. All rights reserved.

\section{Introduction}

Over the four decades or so, nonlinear evolution equations (NEEs) have been the subject of extensive studies in various branches of applied sciences such as fluid mechanics, plasma physics, crystal lattice theory, etc. A special class of analytical solutions, the so-called traveling waves, for NEEs is of fundamental importance because a lot of mathematical-physical models are often described by such a wave phenomena. Thus, the investigation of traveling wave solutions is becoming more and more attractive in nonlinear science nowadays. However, not all equations posed in these fields are solvable. As a result, many new techniques have been successfully developed by a diverse group of mathematicians and physicists, such as inverse scattering method [1], Painleve expansion method [2], Backlund transformation method [3], Hirota's bilinear method [4], symmetry method [5], Jacobi elliptic function method [6], sine-cosine function method [7], tanh-coth function method [8], Weierstrass function method [9], homogeneous balance method [10], F-expansion method [11], Exp-function method [12], $\left(G^{\prime} / G\right)$-expansion method [13], further improved F-expansion method [14], etc. For more direct methods to NEEs, see [15-18]. But, it is sometimes hard and time consuming to solve nonlinear problems with the well-known traditional methods.

Since the nonlinear phenomena is enormously complex, it is still an open problem to develop more powerful methods to extract new exact and explicit solutions for NEEs. Recently, Feng [19-21] proposed an effective method, which is based on the ring theory of commutative algebra, to deal with traveling wave solutions of NEEs. The technique, which is currently called the first integral method, has proven to be a useful tool for finding traveling wave solutions to a variety of nonlinear problems (see, for example, [22-27] and the references therein). The basic idea of the first integral method is to find a polynomial first integral (with polynomial coefficients) of an explicit form to an equivalent autonomous planar system by applying the Division Theorem for two variables in the complex domain. Taking the obtained first integral into account, one may obtain a class of traveling wave solutions to the equation considered in a straightforward manner.

On the other hand, extending some innovative approaches to the investigation of NEEs for traveling wave solutions and obtaining new results seems interesting and helpful to the reader in the physical, chemical, and biological communities. Hence, we find that the applicability of the first integral method to NEEs having distinct physical structures is still an interesting and important research problem.

E-mail address: ismailaslan@iyte.edu.tr 
In the present study, we focus our attention on traveling wave solutions of the regularized long-wave Burgers equation and the Gilson-Pickering equation using the first integral method for the first time. To achieve our goal, we organize the paper as follows: In the next section, we describe the method in brief. In Sections 3 and 4, we analyze our equations. Finally, we state a conclusion in Section 5.

\section{The first integral method}

Let us consider a nonlinear partial differential equation for a function $u=u(x, t)$ in the form

$$
P\left(u, u_{t}, u_{x}, u_{t t}, u_{t x}, u_{x x}, \ldots\right)=0
$$

where $P$ is a polynomial in its arguments and subscripts denote partial derivatives. Using the transformation $u(x, t)=U(\xi)$, $u(x, t)=U(\xi), \xi=k x+w t+\xi_{0}$, where $k, w$, and $\xi_{0}$ are arbitrary constants, we can convert Eq. (1) to an ordinary differential equation (ODE) of the form

$$
P\left(U, k U^{\prime}, w U^{\prime}, k^{2} U^{\prime \prime}, k w U^{\prime \prime}, w^{2} U^{\prime \prime}, \ldots\right)=0,
$$

where $U=U(\xi)$ and the primes denote ordinary derivatives with respect to $\xi$. Next, we introduce a new independent variable

$$
V(\xi)=U(\xi), \quad W(\xi)=U^{\prime}(\xi),
$$

which leads to a system of ODEs of the form

$$
\begin{aligned}
& V^{\prime}(\xi)=W(\xi), \\
& W^{\prime}(\xi)=Q(V(\xi), W(\xi)) .
\end{aligned}
$$

In general, solving planar systems of ODEs like (4) directly is a difficult and challenging task. Hence, based on the qualitative theory of ordinary differential equations [28], if we can find a single first integral to the system (4), then we can reduce Eq. (2) to a first-order integrable ODE. A class of traveling wave solutions can be accordingly derived by solving this first-order differential equation. But, there is no a systematic theory that can tell us how to find the first integrals of Eq. (4), nor is there a logical way for telling us what these first integrals are. In order to present our results in a straightforward manner, let us here introduce the Division Theorem for two variables in the complex domain $\mathbb{C}$.

Theorem 1 (Division Theorem). Suppose that $P(w, z)$ and $Q(w, z)$ are polynomials in $\mathbb{C}[w, z]$ and $P(w, z)$ is irreducible in $\mathbb{C}[w, z]$. If $Q(w, z)$ vanishes at all zero points of $P(w, z)$, then there exist a polynomial $G(w, z)$ in $\mathbb{C}[w, z]$ such that $Q(w, z)=P(w, z) G(w, z)$.

One can prove the Division Theorem either by using the complex theory of several variables [19,29] or by applying the following Hilbert-Nullstellensatz theorem from the ring theory of commutative algebra [30]:

Theorem 2 (Hilbert-Nullstellensatz Theorem). Let $k$ be a field and $L$ an algebraic closure of $k$. Then

(i) Every ideal $\gamma$ of $k\left[X_{1}, \ldots, X_{n}\right]$ not containing 1 admits at least one zero in $L^{n}$.

(ii) Let $\mathbf{x}=\left(x_{1}, \ldots, x_{n}\right)$ and $\mathbf{y}=\left(y_{1}, \ldots, y_{n}\right)$ be two elements of $L^{n}$. For the set of polynomials of $k\left[X_{1}, \ldots, X_{n}\right]$ zero at $\mathbf{x}$ to be identical with the set of polynomials of $k\left[X_{1}, \ldots, X_{n}\right]$ zero at $\mathbf{y}$, it is necessary and sufficient that there exists a $k$-automorphism $s$ of $L$ such that $y_{i}=s\left(x_{i}\right)$ for $1 \leqslant i \leqslant n$.

(iii) For an ideal $\alpha$ of $k\left[X_{1}, \ldots, X_{n}\right]$ to be maximal, it is necessary and sufficient that there exists an $\mathbf{x}$ in $L^{n}$ such that $\alpha$ is the set of $k\left[X_{1}, \ldots, X_{n}\right]$ zero at $\mathbf{x}$.

(iv) For a polynomial $Q$ of $k\left[X_{1}, \ldots, X_{n}\right]$ to be zero on the set of zeros in $L^{n}$ of an ideal $\gamma$ of $k\left[X_{1}, \ldots, X_{n}\right]$, it is necessary and sufficient that there exist an integer $m>0$ such that $Q^{m} \in \gamma$.

Remark 1. The fact that the real field $\mathbb{R}$ is a subfield of the complex field $\mathbb{C}$ is well known. The extension of a given equation in $\mathbb{R}$ to an equation in $\mathbb{C}$ is always possible. If the extended equation has an algebraic curve solution in $\mathbb{C}$, then the intersection of the manifold of this solution and the real plane must be the algebraic curve solution of the original equation in $\mathbb{R}$. Thus, the Division Theorem stated in $\mathbb{C}$ can also be used in $\mathbb{R}[19]$.

\section{The regularized long-wave Burgers equation}

Let us consider the regularized long-wave Burgers equation [31] in the form

$$
u_{t}+u_{x}+12 u u_{x}-\alpha u_{x x}-\beta u_{x x t}=0,
$$

which is a model equation of describing the propagation of surface water in a channel, where $u=u(x, t)$ proportiona 1 to the vertical displacement of the surface of the water from its equilibrium position, the constants $\alpha$ and $\beta$ denote dissipative and dispersive coefficients, respectively. Besides, it represents a balance relation among the dispersion, dissipation and nonlinearity. Recently, Wang and Li [32] investigated Eq. (5) for traveling wave solutions by mean sof the so-called factorization 
technique. Now, to seek for traveling wave solutions of Eq. (5), we first make the transformation $u(x, t)=U(\xi), \xi=x-w t+\xi_{0}$, where $w$ and $\xi_{0}$ are arbitrary constants. Then, integrating the resultant equation once, we get

$$
\beta w U^{\prime \prime}-\alpha U^{\prime}+6 U^{2}+(1-w) U-\beta w d=0,
$$

where the primes denote derivatives with respect to $\xi$ and $d$ is an integration constant. Letting $z=U$ and $y=U^{\prime}$, Eq. (6) can be rewritten as the plane autonomous system

$$
\left\{\begin{array}{l}
\frac{d z}{d \xi}=y, \\
\frac{d y}{d \xi}=r y+b z^{2}+c z+d,
\end{array}\right.
$$

where $r=\alpha \mid \beta w, b=-6 / \beta w$, and $c=(w-1) \mid \beta w$. Suppose that $z=z(\xi)$ and $y=y(\xi)$ are nontrivial solutions of (7). Also, assume that $q(z, y)=\sum_{i=0}^{m} A_{i}(z) y^{i}$ is an irreducible polynomial in the complex domain $\mathbb{C}$ such that

$$
q(z(\xi), y(\xi))=\sum_{i=0}^{m} A_{i}(z) y^{i}=0,
$$

where the polynomials $A_{i}(z)(i=0,1, \ldots, m)$ are relatively prime in $\mathbb{C}$ with $A_{m}(z) \not \equiv 0$. Eq (8) is called a first integral of Eq. (7). We note that $d q / d \xi$ is a polynomial in $z$ and $y$. Thus, $q(z(\xi), y(\xi))=0$ implies that $d q / d \xi=0$. Then, by the Division Theorem, there exists a polynomial $B(z)+C(z) y$ in the complex domain $\mathbb{C}$ such that

$$
\frac{d q}{d \xi}=\frac{\partial q}{\partial z} \frac{d z}{d \xi}+\frac{\partial q}{\partial y} \frac{d y}{d \xi}=(B(z)+C(z) y)\left[\sum_{i=0}^{m} A_{i}(z) y^{i}\right] .
$$

We consider the case $m=2$ of (8). Hence, taking Eqs. (7) and (9) into account, we get

$$
\sum_{i=0}^{2}\left[A_{i}^{\prime}(z) y^{i+1}\right]+\sum_{i=0}^{2}\left[i A_{i}(z) y^{i-1}\left(r y+b z^{2}+c z+d\right)\right]=[B(z)+C(z) y]\left[\sum_{i=0}^{2} A_{i}(z) y^{i}\right] .
$$

Equating the coefficients of $y^{i}(0 \leqslant i \leqslant 3)$ in Eq. (10) leads to the system

$$
\begin{aligned}
& y^{3}: A_{2}^{\prime}(z)=C(z) A_{2}(z), \\
& y^{2}: A_{1}^{\prime}(z)=C(z) A_{1}(z)+(B(z)-2 r) A_{2}(z), \\
& y^{1}: A_{0}^{\prime}(z)=C(z) A_{0}(z)+(B(z)-r) A_{1}(z)-2\left[b z^{2}+c z+d\right] A_{2}(z), \\
& y^{0}: B(z) A_{0}(z)-\left(b z^{2}+c z+d\right) A_{1}(z)=0 .
\end{aligned}
$$

From Eq. (11), we obtain $A_{2}(z)=c_{0} \exp \left(\int C(z) d z\right)$, where $c_{0}$ is an integration constant. Since $A_{2}(z)$ and $C(z)$ are polynomials, we deduce that $C(z)=0$ and $A_{2}(z)$ must be a constant. For simplicity, we can take $A_{2}(z)=1$. Then, Eqs. (12) and (13) reduce to the following equations

$$
\begin{aligned}
& A_{1}^{\prime}(z)=B(z)-2 r, \\
& A_{0}^{\prime}(z)=(B(z)-r) A_{1}(z)-2\left(b z^{2}+c z+d\right) .
\end{aligned}
$$

Balancing the degrees of $A_{0}(z), A_{1}(z)$, and $B(z)$, we can conclude that $\operatorname{deg} B(z)=0$ and $\operatorname{deg} A_{1}(z)=1$. Assuming $B(z)=b_{0}\left(b_{0} \neq 0\right)$ and $A_{1}(z)=a_{1} z+a_{0}\left(a_{1} \neq 0\right)$ in Eq. (15), we obtain $b_{0}=a_{1}+2 r$. Thus, from Eq. (16), we have

$$
A_{0}(z)=-\frac{2}{3} b z^{3}+\frac{1}{2}\left(a_{1}^{2}+r a_{1}-2 c\right) z^{2}+\left[a_{0}\left(a_{1}+r\right)-2 d\right] z+k,
$$

where $k$ is an integration constant. By substituting $A_{0}(z), A_{1}(z)$, and $B(z)$ into Eq. (14) and equating the coefficients of $z^{i}$ $(0 \leqslant i \leqslant 3)$ to zero, we obtain the following system of nonlinear algebraic equations

$$
\begin{aligned}
& z^{3}:-8 b r-10 b a_{1}=0, \\
& z^{2}:-12 c r-6 b a_{0}-12 c a_{1}+6 r^{2} a_{1}+9 r a_{1}^{2}+3 a_{1}^{3}=0, \\
& z^{1}:-24 d r-6 c a_{0}+12 r^{2} a_{0}-18 d a_{1}+18 r a_{0} a_{1}+6 a_{0} a_{1}^{2}=0, \\
& z^{0}:-6 d a_{0}+12 r k+6 a_{1} k=0 .
\end{aligned}
$$

Solving the system (18)-(21) simultaneously, we get the solution set

$$
k=-\frac{15625 c^{3}+3750 c^{2} r^{2}-900 c r^{4}-216 r^{6}}{187500 b^{2}}, \quad d=\frac{625 c^{2}-36 r^{4}}{2500 b}, \quad a_{0}=-\frac{2\left(25 c r+6 r^{3}\right)}{125 b}, \quad a_{1}=-\frac{4 r}{5} .
$$


Now, taking the solution set Eq. (24) into account, Eq. (8) becomes

$$
\begin{aligned}
y^{2} & -\left(\frac{4 r}{5} x+\frac{2 r\left(25 c+6 r^{2}\right)}{125 b}\right) y-\left(\frac{2 b}{3} x^{3}+\frac{1}{2}\left(2 c+\frac{4 r^{2}}{25}\right) x^{2}\right. \\
& \left.+\left(\frac{2 r^{2}\left(25 c+6 r^{2}\right)}{625 b}+\frac{625 c^{2}-36 r^{4}}{1250 b}\right) x+\frac{15625 c^{3}+3750 c^{2} r^{2}-900 c r^{4}-216 r^{6}}{187500 b^{2}}\right)=0,
\end{aligned}
$$

which is a first integral of Eq. (7). Solving Eq. (23), we get

$$
y=\frac{6 b r\left(25 c+6 r^{2}+50 b x\right) \mp \sqrt{3} b\left(25 c+6 r^{2}+50 b x\right)^{3 / 2}}{750 b^{2}} .
$$

Finally, combining Eq. (7) with Eq. (24) and changing to the original variables, we obtain traveling wave solutions to Eq. (5) as

$$
u^{ \pm}(x, t)=-\frac{\alpha^{2}}{25 \beta w}\left(\frac{\exp \left(\frac{\alpha}{5 \beta w}\left(x-w t+\xi_{0}\right)\right)}{1 \pm \exp \left(\frac{\alpha}{5 \beta w}\left(x-w t+\xi_{0}\right)\right)}\right)^{2}+\frac{\alpha^{2}}{50 \beta w}+\frac{1}{12}(w-1),
$$

where $w$ and $\xi_{0}$ remain arbitrary. Our result Eq. (25) can be compared with the result of Wang and Li [32] (Example 4.2 in there) by assigning appropriate values to the arbitrary parameters involved.

\section{The Gilson-Pickering equation}

Next, we consider a nonlinear third-order partial differential equations in the form

$$
u_{t}-\varepsilon u_{x x t}+2 k u_{x}-u u_{x x x}-\alpha u u_{x}-\beta u_{x} u_{x x}=0,
$$

where $\varepsilon, k, \alpha$ and $\beta$ are arbitrary constants. In 1995, Gilson and Pickering [33] introduced Eq. (26) by considering Painlevé analysis. Recently, Wang and Li [32] studied a class of nonlinear evolution equations, which includes (26) as a special case, by proposing a factorization technique. Now, letting $u(x, t)=U(\xi), \xi=x-c t+\xi_{0}$, where $c$ and $\xi_{0}$ are arbitrary constants, in (26), we get

$$
(2 k-c) U^{\prime}+\varepsilon c U^{\prime \prime \prime}-U U^{\prime \prime \prime}-\alpha U U^{\prime}-\beta U^{\prime} U^{\prime \prime}=0 .
$$

We take $\beta=1$ in Eq. (27) for convenience. Then integrating the resultant equation once we obtain

$$
(\varepsilon c-U) U^{\prime \prime}-\frac{\alpha}{2} U^{2}+(2 k-c) U=g,
$$

where the primes denote derivatives with respect to $\xi$ and $g$ is an integration constant. Taking $z=U$ and $y=U^{\prime}$ in Eq. (28), we get the equivalent two-dimensional Hamiltonian system

$$
\left\{\begin{array}{l}
\frac{d z}{d \xi}=y \\
\frac{d y}{d \xi}=\frac{(2 k-c) z-\frac{\alpha}{2} z^{2}-g}{z-\varepsilon c}
\end{array}\right.
$$

with Hamiltonian function

$$
H(z, y)=\frac{1}{4}\left(2 y^{2}+z(4 c-8 k+(2 c \varepsilon+z) \alpha)+2(2 g+c \varepsilon(2 c-4 k+c \varepsilon \alpha)) \operatorname{In}(z-c \varepsilon)\right)=h,
$$

where $h$ is a constant. We make the transformation $d \xi=(z-\varepsilon c) d \eta$ in (29) to avoid the singular line $z=\varepsilon c$ for a while. Thus, system (29) becomes

$$
\left\{\begin{array}{l}
\frac{d z}{d \eta}=(z-\varepsilon c) y \\
\frac{d y}{d \eta}=(2 k-c) z-\frac{\alpha}{2} z^{2}-g
\end{array}\right.
$$

Then the system (29) has the same topological portraits as the system (31) except for the straight line $z=\varepsilon c$. For the new system (31), $z=\varepsilon c$ is its invariant straight line solution. We consider the case $m=1$ of (8). From now on, we will omit some of the details because the procedure is the same. Then, by equating the coefficients of $y^{i}(0 \leqslant i \leqslant 2)$ on both sides of (9), we have

$$
\begin{aligned}
& y^{2}:(z-\varepsilon c) A_{1}^{\prime}(z)=C(z) A_{1}(z), \\
& y^{1}:(z-\varepsilon c) A_{0}^{\prime}(z)=C(z) A_{0}(z)+B(z) A_{1}(z), \\
& y^{0}: B(z) A_{0}(z)=\left[(2 k-c) z-\frac{\alpha}{2} z^{2}-g\right] A_{1}(z) .
\end{aligned}
$$

Since $A_{1}(z)$ and $C(z)$ are polynomials, from Eq. (32), we deduce that $C(z)=0$ and $A_{1}(z)$ must be a constant. For simplicity, we can take $A_{1}(z)=1$. Then, Eq. (33) indicates that $\operatorname{deg} B(z) \leqslant \operatorname{deg} A_{0}(z)$. Thus, from Eq. (34), we conclude that 
$\operatorname{deg} B(z)=\operatorname{deg} A_{0}(z)=1$. Assuming $A_{0}(z)=a_{1} z+a_{0}\left(a_{1} \neq 0\right)$ and $B(z)=b_{1} z+b_{0}\left(b_{1} \neq 0\right)$ in Eq. (33), we get $b_{1}=a_{1}$ and $b_{0}=-c \varepsilon a_{1}$. Substituting $A_{0}(z)$ and $B(z)$ into Eq. (34) and setting the coefficients of $z^{i}(0 \leqslant i \leqslant 2)$ to be zero, we derive a system of nonlinear algebraic equations for $a_{0}, a_{1}, c$, and $g$. Solving the resultant system simultaneously, we get the solution set

$$
g=-\frac{1}{2} c \varepsilon(2 c-4 k+c \alpha \varepsilon), \quad a_{0}=\mp \frac{i(2 \sqrt{2} c-4 \sqrt{2} k+\sqrt{2} c \alpha \varepsilon)}{2 \sqrt{\alpha}}, \quad a_{1}=\mp \frac{i \sqrt{\alpha}}{\sqrt{2}} .
$$

where $c$ remains arbitrary. Using the condition (35) in (8), we obtain

$$
y= \pm \frac{i \sqrt{\alpha}}{\sqrt{2}} x \pm \frac{i(2 \sqrt{2} c-4 \sqrt{2} k+\sqrt{2} c \alpha \varepsilon)}{2 \sqrt{\alpha}} .
$$

Combining Eq. (29) with Eq. (36) and changing to the original variables, we find traveling wave solutions to Eq. (26) (with $\beta=1)$ as

$$
\begin{aligned}
& u_{1}^{ \pm}(x, t)=\frac{4 k-c(2+\alpha \varepsilon)}{\alpha}+\frac{1}{\alpha} \exp \left( \pm \frac{i \sqrt{\alpha}}{\sqrt{2}}\left(x-c t+\xi_{0}\right)\right), \quad \alpha>0, \\
& u_{2}^{\mp}(x, t)=\frac{4 k-c(2+\alpha \varepsilon)}{\alpha}+\frac{1}{\alpha} \exp \left(\mp \frac{\sqrt{-\alpha}}{\sqrt{2}}\left(x-c t+\xi_{0}\right)\right), \quad \alpha<0,
\end{aligned}
$$

where $c$ and $\xi_{0}$ remain arbitrary. Our results (37) and (38) can be compared with the result of Wang and Li [32] (Example 4.1 in there) by assigning special values to the arbitrary parameters involved.

Remark 2. We analyzed our equations by assuming $m=1,2$ in Eq. (8), respectively. The discussion becomes more complicated for the cases $m=3,4$ since the hyper-elliptic integrals, the irregular singular point theory, and the elliptic integrals of the second kind are involved. We do not need to consider the case $m \geqslant 5$ because of the fact that an algebraic equation with the degree greater than or equal to 5 is generally not solvable.

\section{Conclusion}

Searching for first integrals of nonlinear ODEs is one of the most important problem since they permit us to solve a nonlinear differential equation by quadratures. Applying the first integral method, which is based on the ring theory of commutative algebra, we established some traveling wave solutions to the regularized long-wave Burgers equation and the GilsonPickering equation. We observed that the first integral method, for discovering first integrals, can be applied to NEEs which can be converted to a second-order ODE through the traveling wave transformation. From our results, we can see that the technique used in this paper is very effective and can be steadily applied to nonlinear problems, especially for the ones including non-integrable equations, arising in applied mathematics.

\section{Acknowledgment}

The author thanks the anonymous referees for the valuable and useful suggestions.

\section{References}

[1] M.J. Ablowitz, H. Segur, Solitons and Inverse Scattering Transform, SIAM, Philadelphia, 1981.

[2] J. Weiss, M. Tabor, G. Carnevale, The Painleve property for partial differential equations, J. Math. Phys. 24 (1983) 522-526.

[3] R.M. Miura, Backlund Transformation, Springer-Verlag, New York, 1973.

[4] R. Hirota, The Direct Method in Soliton Theory, Cambridge University Pres, Cambridge, 2004.

[5] G.W. Bluman, S. Kumei, Symmetries and Differential Equations, Springer-Verlag, New York, 1989.

[6] G.T. Liu, T.Y. Fan, New applications of developed Jacobi elliptic function expansion methods, Phys. Lett. A. 345 (2005) 161-166.

[7] C. Yan, A simple transformation for nonlinear waves, Phys. Lett. A. 224 (1996) 77-84.

[8] W. Malfliet, W. Hereman, The tanh method I: exact solutions of nonlinear evolution and wave equations, Phys. Scr. 54 (1996) $563-568$.

[9] Z.Y. Yan, An improved algebra method and its applications in nonlinear wave equations, Chaos Solitons Fract. 21 (2004) $1013-1021$.

[10] M.L. Wang, Exact solutions for a compound KdV-Burgers equation, Phys. Lett. A 213 (1996) 279-287.

[11] M.A. Abdou, The extended $F$-expansion method and its application for a class of nonlinear evolution equations, Chaos Solitons Fract. 31 (2007) $95-104$.

[12] J.H. He, X.H. Wu, Exp-function method for nonlinear wave equations, Chaos Solitons Fract. 30 (2006) 700-708.

[13] M. Wang, X. Li, J. Zhang, The $\left(G^{\prime} \mid G\right)$-expansion method and travelling wave solutions of nonlinear evolution equations in mathematical physics, Phys. Lett. A. 372 (2008) 417-423.

[14] D. Wang, H.Q. Zhang, Further improved F-expansion method and new exact solutions of Konopelchenko-Dubrovsky equation, Chaos Solitons Fract. 25 (2005) 601-610.

[15] E. Fan, Soliton solutions for a generalized Hirota-Satsuma coupled KdV equation and a coupled MKdV equation, Phys. Lett. A. 282 (2001) 18-22.

[16] D.S. Wang, A systematic method to construct Hirota's transformations of continuous soliton equations and its applications, Comput. Math. Appl. 58 (2009) $146-153$.

[17] D.S. Wang, H.B. Li, Elliptic equation's new solutions and their applications to two nonlinear partial differential equations, Appl. Math. Comput. 188 (2007) 762-771.

[18] D.S. Wang, Z. Yan, H. Li, Some special types of solutions of a class of the ( $N+1)$-dimensional nonlinear wave equations, Comput. Math. Appl. 56 (2008) 1569-1579. 
[19] Z. Feng, X. Wang, Explicit Exact Solitary Wave Solutions for the Kundu Equation and the Derivative Schrödinger Equation, Phys. Scr. 64 (2001) 7-14.

[20] Z. Feng, On explicit exact solutions to the compound Burgers-KdV equation, Phys. Lett. A. 293 (2002) 57-66.

[21] Z. Feng, The first-integral method to study the Burgers-Korteweg-de Vries equation, J. Phys. A: Math. Gen. 35 (2002) $343-349$.

[22] Z. Feng, S. Zheng, D.Y. Gao, Traveling wave solutions to a reaction-diffusion equation, Z. Angew. Math. Phys. 60 (2009) $756-773$.

[23] Z. Feng, R. Knobel, Traveling waves to a Burgers-Korteweg-de Vries-type equation with higher-order nonlinearities, J. Math. Anal. Appl. 328 (2007) $1435-1450$.

[24] X. Deng, Traveling wave solutions for the generalized Burgers-Huxley equation, Appl. Math. Comput. 204 (2008) $733-737$.

[25] Naranmandula, K.X. Wang, New explicit exact solutions to a nonlinear dispersive-dissipative equation, Chin. Phys. 13 (2004) $139-143$.

[26] K.R. Raslan, The first integral method for solving some important nonlinear partial differential equations, Nonlinear Dyn. 53 (2006) $281-286$.

[27] S. Abbasbandy, A. Shirzadi, The first integral method for modified Benjamin-Bona-Mahony equation, Commun. Nonlinear Sci. Numer. Simulat. 15 (2010) 1759-1764.

[28] T.R. Ding, C.Z. Li, Ordinary Differential Equations, Peking University Press, Peking, 1996.

[29] Z. Feng, Algebraic curve solution for second-order polynomial autonomous systems, Electron J. Linear Algebra 8 (2001) $14-25$.

[30] N. Bourbaki, Commutative Algebra, Addison-Wesley, Paris, 1972.

[31] J.L. Bona, W.G. Pritchard, L.R. Scott, An evaluation of a model equation for water waves, Philos. Trans. R. Soc. Lond. Ser. A 302 (1981) 457-510.

[32] D.S. Wang, H. Li, Single and multi-solitary wave solutions to a class of nonlinear evolution equations, J. Math. Anal. Appl. 343 (2008) 273-298.

[33] C. Gilson, A. Pickering, Factorization and Painlevé analysis of a class of nonlinear third-order partial differential equations, J. Phys. A: Math. Gen. 28 (1995) 2871-2888. 